\title{
Simulation and Study on Plate-Type Total Pressure Distortion Simulators
}

\author{
Zhipeng Li, Guowang Zhang and Chao Lu \\ AECC Shenyang Engine Research Institute, No. 1, Wanlian Road, Shenhe District, Shenyang, China
}

\begin{abstract}
In China, plate-type total pressure distortion simulators are widely used for carrying on inlet total pressure test of compressing systems of aeroengine. In this article modeling and simulating were imposed on this type of simulators. According to the simulation results, details of distortion field configuration formed on the test inlet aerodynamic interface plane (AIP) as well as total pressure isoline distributions were described, data on the relative practical total-pressure survey points were picked out meanwhile. Furthermore, relationships between blocking ratio and area of low-pressure zone on AIP along with steady distortion descriptor were searched for. Possible reference to future design of irregular plate-type totalpressure distortion simulator from this time of simulation is discussed.
\end{abstract}

Keywords-plate-type total pressure distortion simulator; distortion field; blocking ratio; distortion descriptor

\section{INTRODUCTION}

Compressing system is one of the three key components of aeroengine with characteristics of high pressure, high revolution speed and high temperature, both design and test are extremely difficult. In a operation, since there is flow disturbance from intake passage ahead and inner, intake total pressure distortion is frequently occurs on the inlet of compressing system, which is one of the most common type of intake distortion. The test aiming to this type of distortion is called a total pressure distortion test, which is a very important process to determine the stability of compressing system. In aeroengine industry in China, plate-type total pressure simulators are generally used. By altering the blocking ratio of the simulator, various inlet total pressure distortion descriptors are able to be obtained. Furthermore, calculating the distortion sensitivity can be done after gaining the loss in the performance of test article to determine its anti-distortion ability $(1,2)$.

Having been widely used, the plate-type total pressure distortion simulators cannot describe details of the whole distortion field because of test restrictions, especially the number of survey points, which leads to a small amount of the whole inlet measurement plane to be applied. To solve the problem, in this article computational fluid mechanics (CFD), which has been greatly developed and taken into application in recent years, was introduced. By modeling and simulation, the distortion fields formed on the test article AIP by simulators with different blocking ratios were obtained to describe a complete field distribution, and validate the reliability by comparing key test parameters.

\section{The STRUCTURE OF Plate-TYPe Distortion SIMULATOR AND PARAMETERIZED DESCRIPTION OF DISTORTION FIELD}

A plate-type total distortion simulator includes a straight pipe and a lunette-shaped plate inside the pipe, as is shown in Figure I.

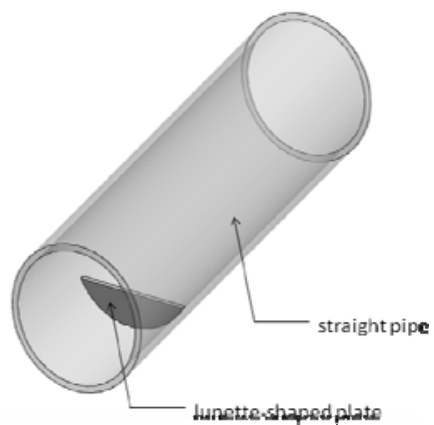

FIGURE I. THE STRUCTURE OF A PLATE-TYPE TOTAL DISTORTION SIMULATOR

Before distortion test, the facility is assembled between test article inlet and the intake system of test rig, see Figure II. In this way, the lunette-shape plate will offer blocking effect to the intake of test article, leading to an asymmetrical total pressure distribution at inlet. In a test the total pressure distortion descriptor is frequently used to scale the level of distortion, which includes two components: steady-state distortion descriptor and time-variant distortion descriptor. This simulation just refers to steady-state distortion descriptor and only the expression is given out for time-variant descriptor but without deep study since merely stationary calculation has been done. Actually, as previous study shows, the changing rule of time-variant distortion descriptor is able to be understood through steady-state one since there is similar quantum between them (3).

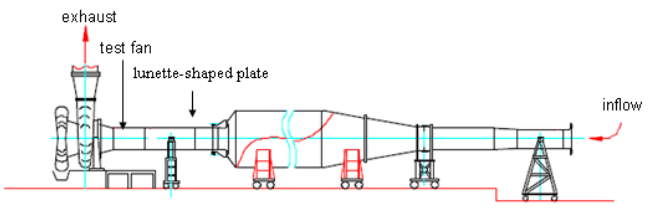

FIGURE II. A TEST RIG WITH PLATE-TYPE SIMULATOR 
The expressions of steady-state distortion descriptor and time-variant distortion descriptor are given below:

Steady-state distortion descriptor $\Delta \sigma_{0}$ is defined as:

$$
\Delta \sigma_{0}=\left(1-\sigma_{0} / \sigma_{c p}\right)
$$

here $\sigma_{0}$ is the average value obtained from integral of total pressure along radial and circumference on low-pressure of AIP, and $\sigma_{c p}$ is the average value of total pressure ones gained by all survey points on AIP.

Time-variant distortion descriptor $\mathcal{E}$ is defined as:

$$
\varepsilon=\frac{\sum_{1}^{m} \frac{\sqrt{\frac{1}{t} \int_{0}^{t}\left[P_{c p}^{*}-P_{i}^{*}\right]^{2} \cdot d t}}{P_{c p}^{*}}}{m}
$$

Here $t$ is time interval, $m$ is the total number of timevariant instruments, $P_{c p}^{*}$ is average value of total pressure at a certain point during an interval time of $t, P_{i}^{*}$ is a instantaneous value of total pressure at a certain point during an interval time of $t$.

Generally speaking, in a test six total pressure probes with six survey points each are assembled on AIP, the survey points of each probe are ranged with equal-torus rule, as what was shown in Figure III.

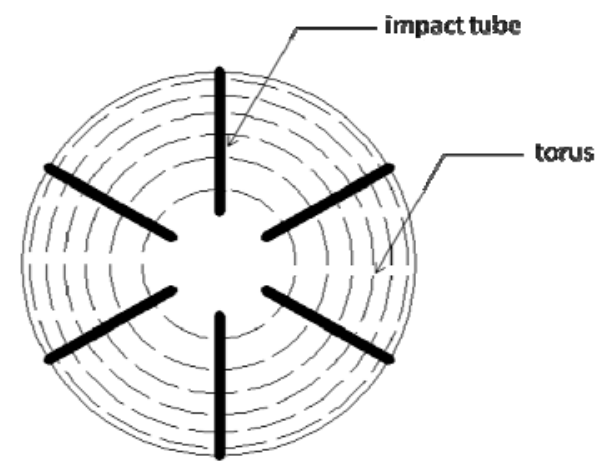

FIGURE III. IMPACT TUBE ASSEMBLED ON AIP

At present a complete method for inlet total pressure distortion test has been buildup and a series of practices has been done (4), the test contents and results are not deeply referred in this article.

In this article, the way of getting data to be solved is the same as what had been done in the test, namely to pick up total pressure values at practical locations of survey points. Detailed analysis was carried out in the following chapter.

\section{MESHING AND INITIAL BOUNDARY CONDITIONS SETUP}

Since there are no differences among the structures of each plate-type distortion simulators except for blocking ratio, hence in this article only simulator with $20 \%$ blocking ratio is applied as an example to give out the conditions of mesh computing and initial boundary conditions setup.

Getting mesh computing for the structure of simulator which was shown in Figure 1, the software of the mesh computing is ANSYS ICME. To get a good quality of mesh and decrease its amount, furthermore increase the efficiency of simulating convergence, 3D structural mesh (or hexahedral mesh) is applied, combined with boundary layer mesh near the wall. The computing mesh condition is shown (only mesh for inlet is shown) in Figure IV:

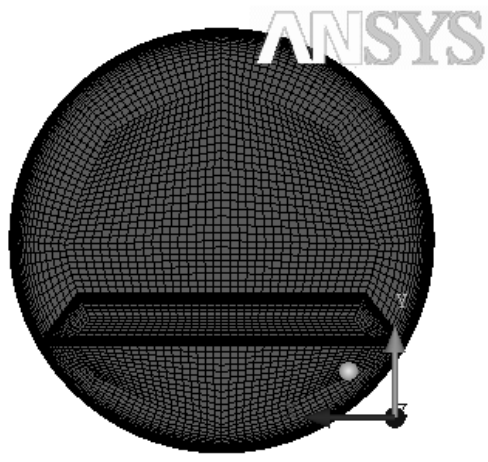

FIGURE IV. MESHING CONDITIONS

According to data statistic from ICME, the total number of mesh elements is about 740,000, while the nodes are about 72,000 , the ratio of elements to nodes is nearly 1 , with the lowest quality 0.45 , which satisfy the requirement of simulation.

The initial boundary condition setup is shown in Table 1.

TABLE I.

INITIAL BOUNDARY CONDITION SETUP

\begin{tabular}{|l|l|}
\hline \multicolumn{1}{|c|}{ Name of the boundary } & \multicolumn{1}{c|}{ Boundary type and parameters } \\
\hline inlet & Inlet, total pressure \\
\hline Outlet( namely AIP) & Outlet, velocity \\
\hline $\begin{array}{l}\text { Inner wall of the straight pipe and } \\
\text { outer wall of the plate }\end{array}$ & No slip wall, smooth wall \\
\hline
\end{tabular}

What should be pointed out is that in a distortion test, the temperature of flow in the simulator has no change, for this reason in the simulation no heat transformation is considered.

\section{Simulation Result Analysis}

Standard $k-\varepsilon$ model is selected as turbulence model. There is a study on effect of different turbulence models to the results, so in this article no more discuses about this issue is present. The results gained from calculation are solved in the post-solving module of ANSYS CFX.

First the total pressure distribution on AIP of the simulators with different blocking ratios are shown in Figure5 (a) to (e) 


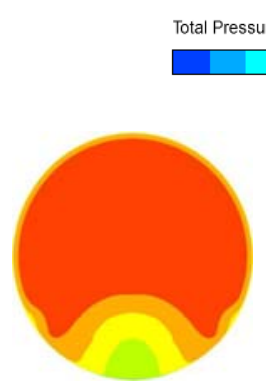

(a)

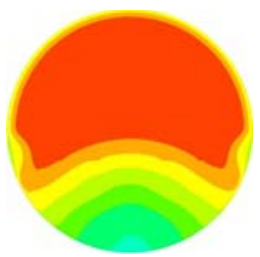

(c)

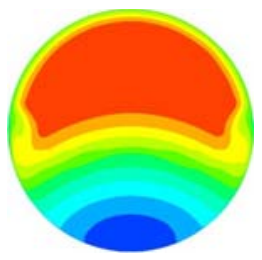

(e)

FIGURE V TOTAL PRESSURE DISTRIBUTION ON AIP OF THE SIMULATORS WITH DIFFERENT BLOCKING RATIOS

It is declared that AIP is at a location 2.5 times of pipe diameter along the axis downstream, where satisfies the relative standards of inlet total pressure distortion test.

From Figure $\mathrm{V}$ it can be seen that the structure of inlet distortion field is accordant with the location and blocking ratio of the simulator plate, blocking ratio is the key factor to influent the level of distortion. Besides, distribution of lowpressure zone has a certain regular as well: firstly, looking at the total pressure changes from low to high, isolines changes just like "water wave", namely from a "core" to diffuse around. The diffusing effect is influenced by the "gathering" effect from inner wall of the straight pipe and weakens gradually, leading to a steady boundary of high-pressure zone, finally formed a vaulted high-pressure zone. Secondly, the boundary layer of the straight pipe shows a certain level of influence to the high-pressure zone, and the influence gets larger with a bigger blocking ratio. The survey points in the high-pressure zone near the wall will get a smaller data respectively.

The distortion field gained from simulation is eudipleural, which is slightly different from what was gained from the test. A possible reason may be that the simulation considered no influence of rotor downstream to the field on AIP, latter study may validate it by giving a swirl velocity field at outlet or processing a complete machine test, but not in this article.

Figure VI gives the relationship curve between the area of low-pressure zone of AIP and different blocking ratios:

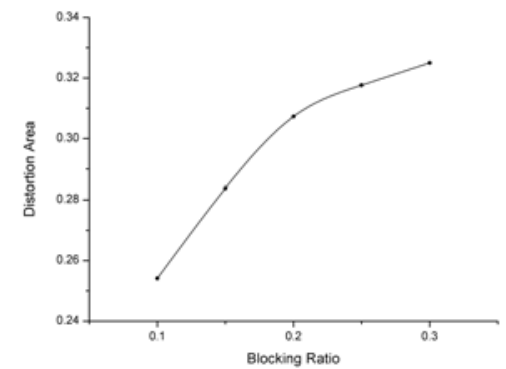

FIGURE VI. THE RELATIONSHIP CURVE BETWEEN THE AREA OF LOW-PRESSURE ZONE OF AIP AND DIFFERENT BLOCKING RATIOS

From Figure IV it can be observed that the curve showing the relationship between blocking ratio and distortion area can be divided into two line segments with different slop, the cutoff point is the one whose blocking ratio is 0.2 . When blocking ratio is smaller than 0.2 , the distortion area increases with a higher speed when blocking ratio rises, while the speed slows down when the blocking ratio is larger than 0.2.

The curve shows the relationship between steady-state distortion descriptor and the relative blocking ratio is shown in Figure7, the total pressure values come from each cases, the final results of steady-state distortion descriptors were calculated by the same software used the test.

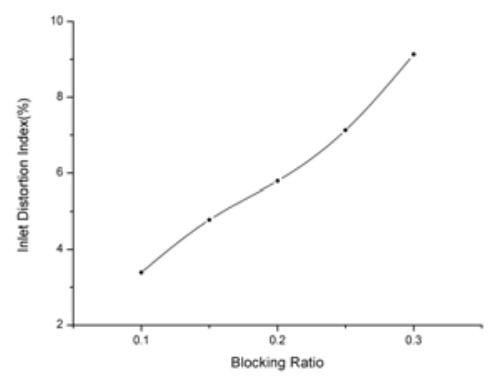

FIGURE VII. THE RELATIONSHIP BETWEEN STEADY-STATE DISTORTION DESCRIPTOR AND THE RELATIVE BLOCKING RATIO

From Figure VII it can be seen that the blocking ratio has a closely linear relationship with inlet steady-state total pressure distortion descriptor. Making block ratio $\mathrm{br}$, then according to the relationship curve in Figure7 an expression between descriptor and blocking ratio can be fitted below:

$$
\Delta \sigma_{0}=27.66 b r+0.512
$$

This expression can be considered of being used to select the blocking ratio of distortion simulator.

The curves in Figure6 and Figure7 match well with some studies and test data which have been done previously [6-11], indicate a high reliability of this simulation. 


\section{CONCLUSIONS AND PROSPECTS}

In this article the widely used plate-type distortion simulators are studied. Applying CFD the total pressure distortion fields on AIP are gained from the simulators with different blocking ratios. By analyzing the simulation data and contours the following conclusions are given out:

1. The distortion fields formed on AIP are corresponding to the location of the plates as well as blocking ratios, blocking ratio is the key factor of constructing the distortion field in a plate distortion test;

2. Total pressure isolines distribute as "water wave", at the downside place on AIP where corresponding to the plate there is a minimal total pressure zone. And taking the zone as a core, total pressure isolines diffuses to the upper side of AIP, and with gathering effect the high-pressure zone finally shows a vaulted shape;

3. The parameters taken near the wall of the straight pipe are smaller because of the effect of boundary layer, and larger blocking ratio leads to a more instinct influence;

4. The area of low-pressure zone on AIP increases with the blocking ratio rising in two steps, the cutoff point is 0.2 of blocking ratio, when the ratio is larger than 0.2 , the increasing speed slows down;

5. The total pressure distortion descriptor has a increasing linear relationship with blocking ratio, in this article a expression between the two was given.

Because of the restrict of research stage and computing resource, in this article no more simulation combined simulator and test article is carried out, leading to a deviation between test result and simulation one. However, through the simulation, detailed flow field data on AIP can be obtained, the relationship between the area of low-pressure zone and blocking ratio, as well as the one between distortion descriptor and blocking ratio are analyzed after, which is good to select a proper simulator for the following distortion test. Besides, the structure of plate-type simulator is much too simple to provide a more complex distortion field formed in a practical environment, thus design of irregular plate-type simulators has to be done. In China the researchers are lake of experience of doing this. By referring to the distortion effect of plate-type simulator, researchers may get some illuminations.

\section{REFERENCES}

[1] Haibo Xue, "Numerical Simulation and Parametric Analysis of Flow Field Distortion behind Inlet Flat Baffle”, Nanjing University of Aeronautics and Astronautics, Nanjing, December, 2008;

[2] Sun Peng, Gao Haiyang, Zhong Jinjun, Numerical Simulation of NonUniform Flow Field Structure Behind Classic Flat Baffle, Journal of Propulsion Technology, Beijing, Vol. 34 No. 2, 2013, pp. 173-180;

[3] Li Liang, Hu Jun, Wang Zhiqiang, Experimental study of inlet totalpressure distortion on four kinds of flat baffles, Journal of Aerospace Power, Beijing, Vol. 24 No. 4, 2009, pp. 925-930;

[4] Wu Hui, Zhang Guo-Wang, Yang Ming-Sui, Experiment on inlet pressure distortion of high-pressure compressor, Journal of Aerospace Power, Beijing, Vol. 29 No. 7, 2014, pp. 1660-1666

[5] Gan Tian,Wang Rugen,Zhang Jie, Numerical Simulation of Inlet Distortion with Interceptor with Different Turbulence Models, Journal of Propulsion Technology, Beijing, Vol.35 No.7, 2014, pp. 891-896;
[6] Liu Daxiang, Ye Peiliang, Assessment Manual on Inlet Flow Field Distortion of Russian Aeroengine, Gas Turbine Experiment and Research, Chengdu, $3^{\text {rd }}$ edition, 1994, pp. 1-10;

[7] Jiang Yong, Zhang Faqi, Zhang Bailing, The Inserted Board Air Inlet Flow Steady Pressure Distortion Field Estimate Based on Experiment, Journal of Air Force Engineering University (Natural Science Edition), Xi'an, Vol. 8 No. 2, 2007, pp. 1-3;

[8] Gu Wei-Qun, Qin Min, Chen Jian-Min, Design and Test of Movable Baffle Pressure Distortion Generator, Gas Turbine Experiment and Research, Chengdu, $1^{\text {st }}$ edition, 2006, 54-62;

[9] Li Wenfeng, Ma Lidong, Wang Yongsheng, Method and Experimenta Facility for Assessing Pressure Distortion of a Certain Turbofan Engine, Journal of Northwestern Polytechnical University, Xi'an, Vol. 21 No. 5, 2003, pp. 540-543;

[10] Cheng Bangqin, Tao Zengyuan, Li Jun, Aerodynamic stability analysis of inlet total pressure distortion for turbofan, Journal of Propulsion Technology, Beijing, Vol. 24 No. 1, pp. 21-23;

[11] Luo Biaoneng, Investigation of Compressor Stability with Distortion by Inlet Insert-board, Nanjing University of Aeronautics and Astronautics, Nanjing, January, 2006. 